\title{
Measurements of vector boson plus jets from ATLAS
}

\author{
Yoichi Ninomiya $^{1, a}$ on behalf of the ATLAS Collaboration \\ ${ }^{1}$ International Center for Elementary Particle Physics, The University of Tokyo, 113-0033, Japan
}

\begin{abstract}
Measurements of single $W, Z$ boson production in association with jets probe QCD in multi-scale environment. Measurements of $W+$ jet and $Z+$ jet production and their ratio, extending to high jet multiplicities, and a large set of kinematic distributions are presented for $7 \mathrm{TeV}$ data. The results are compared to the predictions of modern Monte Carlo generators. The production of vector bosons plus heavy flavors is sensitive to the $c$ and $b$ quark parton distribution functions (PDFs) and gluon splitting effects. Measurements of $W+c$ and $Z+b(b)$ are reported and compared to predictions based on various PDFs in 4 and 5 flavour schemes. An overview of the results is given.
\end{abstract}

\section{Introduction}

The production of vector bosons in association with jets is an important probe for perturbative quantum chromo dynamics (QCD). These processes are also non-negligible backgrounds for new physics searches and Higgs boson measurements. In addition, measurements of vector boson plus heavy flavour production can constrain the parton distribution function (PDF) of the proton. These processes are studied using $4.6 \mathrm{fb}^{-1}$ of proton-proton collision data at $\sqrt{s}=7 \mathrm{TeV}$ collected with the ATLAS detector [1] at the Large Hadron Collider (LHC) [2]. Recently, there have been advances in the theoretical framework for the calculation at next-to-leading order (NLO) of vector boson plus jets with up to 5 jets in the final state [3]. NLO matrix element calculations have also been available for vector boson associated $b$ quark production at parton level [4-7]. The measurements on these vector boson plus jets are compared with a number of new theoretical predictions. In this paper, results on $W+$ jets [8], $Z+$ jets [9], the ratio of $W / Z+$ jets [10], $W+b$ jets [11], $Z+b$ jets [12], and $W+c$ [13] productions are summarized.

\section{$2 W+$ jets production}

The cross section for the $W(\rightarrow \ell v)+$ jets process is measured as a function of jet multiplicity, transverse momentum $\left(p_{T}\right)$, rapidity of jets, the sum in $p_{T}$ of the jets and lepton, angular separation and invariant mass of the leading two jets. Differential jet cross sections are obtained for $p_{T}$ of jets up to $1 \mathrm{TeV}$ and for multiplicities up to 7 jets. Figures 1 and 2 show leading jet $p_{T}$ distribution for the case of $W+\geq 1$ jet and $H_{T}$ distribution for the case of $W+\geq 3$ jets. $H_{T}$ is the scalar $p_{T}$ sum of the lepton and the jets in the event. The data is compared to BLACKHAT+SHERPA [14-16], ME+PS@NLO $[17,18]$ and HEJ [19] for NLO predictions, LoopSim [20] for NNLO predictions, and ALPGEN [21]

\footnotetext{
ae-mail: ninomiya@icepp.s.u-tokyo.ac.jp
} 
and SHERPA $[17,18]$ for LO predictions. For the jet transverse momentum distribution, ALPGEN, SHERPA and ME+PS@NLO are in reasonable agreement with the data, although ME+PS@NLO shows some deviations at low jet $p_{T}$. BLACKHAT+SHERPA and LoopSim show disagreement at high jet $p_{T}$. These theoretical predictions are in good agreement with the data for the $H_{T}$ distribution except SHERPA prediction; it tends to be above the data in the high $H_{T}$ region.
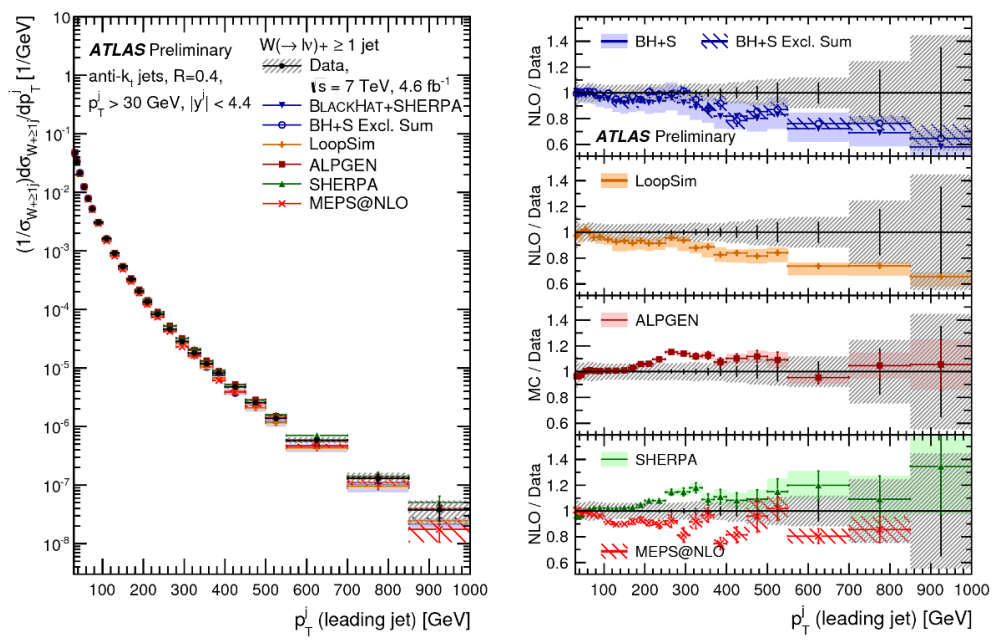

Figure 1. $W+$ jets cross section as a function of the leading jet $p_{T}$ for $N_{\text {jets }} \geq 1$ events. The statistical uncertainty is shown by the vertical bars, and the combined statistical and systematic uncertainties are shown by the hashed regions. The left-hand figure shows the differential cross sections and the right-hand figure shows the ratios of the predictions to the data [8].
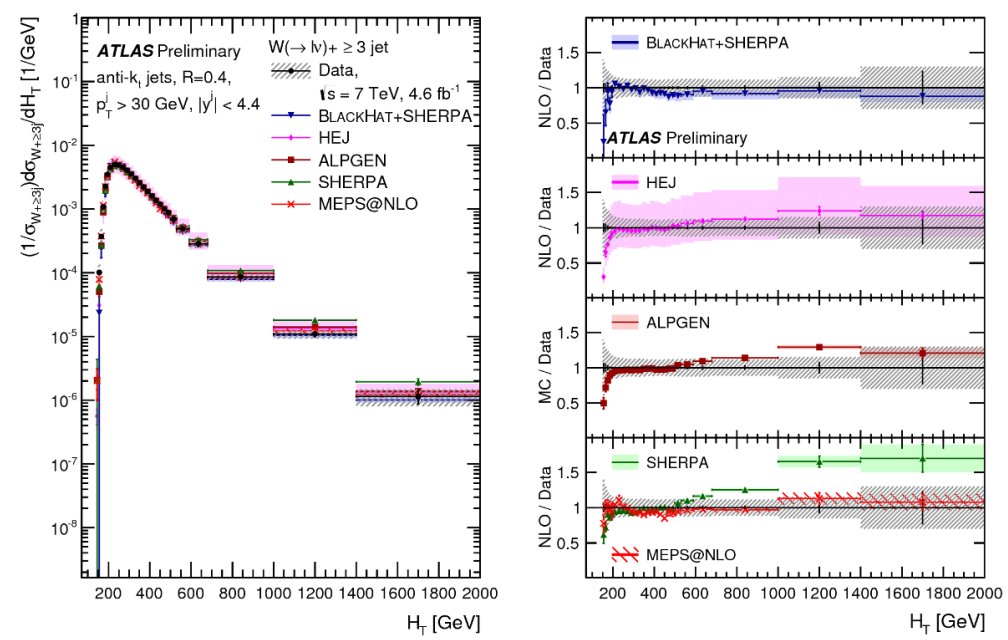

Figure 2. $W+$ jets cross section as a function of $H_{T}$ for $N_{\text {jets }} \geq 3$ events. The statistical uncertainty is shown by the vertical bars, and the combined statistical and systematic uncertainties are shown by the hashed regions. The left-hand figure shows the differential cross sections and the right-hand figure shows the ratios of the predictions to the data [8]. 


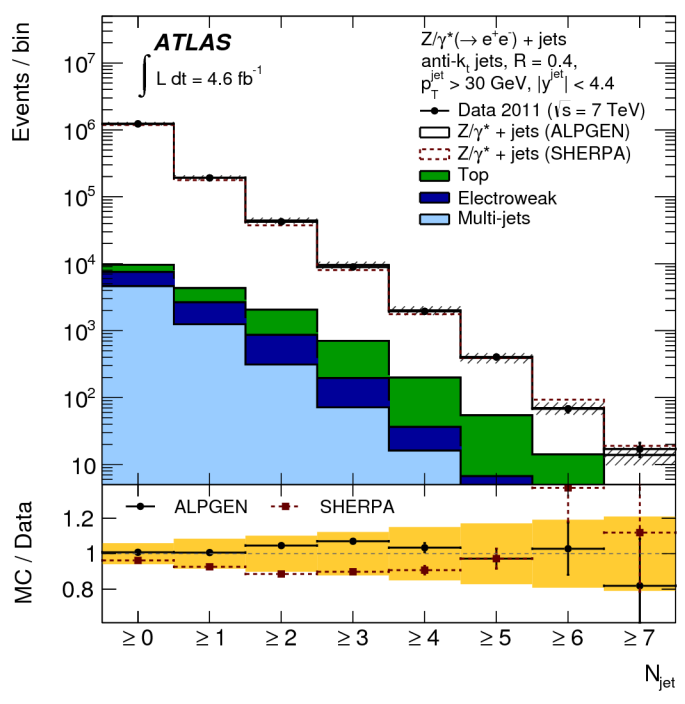

Figure 3. Number of events observed in the data and $\mathrm{MC}$ that pass the $Z(\rightarrow e e)+$ jets selections as a function of the inclusive jet multiplicity. The vertical bars show the statistical uncertainties, and the hatched bands show the combined statistical and systematic uncertainties. The bottom panel shows the corresponding MC/data ratio. The yellow band corresponds to the total uncertainties. The error bar shows only statistical uncertainties on the MC/data ratio [9].

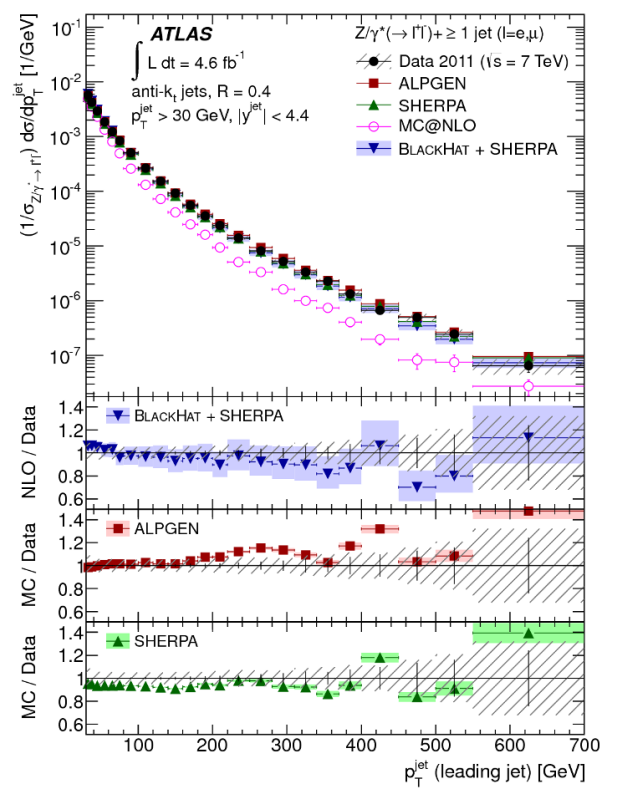

Figure 4. $Z(\rightarrow \ell \ell)+\geq 1$ jets cross section as a function of the leading jet $p_{T}$. The error bars indicate the statistical uncertainty on the data, and the hatched (shaded) bands the combined statistical and systematic uncertainties on the data (prediction) [9].

\section{$3 Z+$ jets production}

The background in the $Z(\rightarrow \ell \ell)+$ jets event selections is smaller than that in the $W+$ jets selections. However the precision of the measurement is lower than the one of $W(\rightarrow \ell v)+$ jets due to its lower cross section. Figure 3 shows number of events that pass the $Z+$ jets selections as a function of the inclusive jet multiplicity. The data is compared with ALPGEN and SHERPA. The data is in good agreement with these Monte Carlo predictions. Figure 4 shows the cross section as a function of leading jet $p_{T}$ for events with at least one jet in the final state. The cross sections are normalized to the inclusive $Z(\rightarrow \ell \ell)$ cross section. The theoretical NLO predictions are consistent with the data, although MC@NLO [22] shows deviation from the data by one order of magnitude in large $p_{T}$ regions.

\section{The ratio of $W / Z+$ jets production}

The measurements of the cross section ratio $\left(R_{\text {jets }}\right)$ of $W+$ jets to $Z+$ jets are complementary tests of perturbative QCD to the individual cross section measurements of $W+$ jets and $Z+$ jets. Some experimental uncertainties and effects from non-perturbative processes, such as hadronisation and multi-parton interactions, are greatly reduced in the ratio. This allows precision comparisons with state-of-the-art Monte Carlo simulations and NLO perturbative QCD calculations. Figure 5 shows the $R_{\text {jets }}$ as a function of inclusive jet multiplicity. The theoretical predictions describe the data fairly well, 


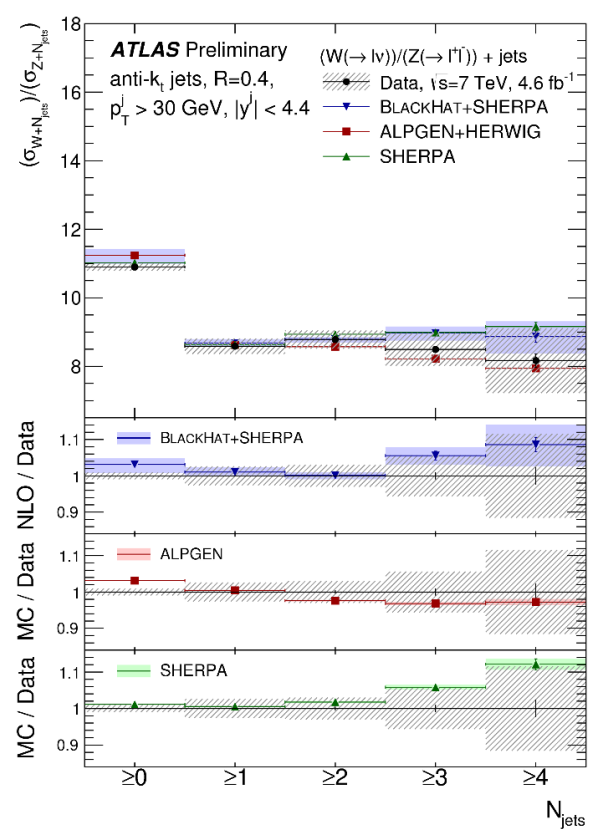

Figure 5. $R_{\text {jets }}$ as a function of inclusive jet multiplicity. The electron and muon channel measurements are combined. Ratios of the simulation to the data are shown in the lower panels. Vertical error bars show the respective statistical uncertainties. The hatched error band shows statical and systematic uncertainties added in quadrature for the data. The solid error bands show statistical uncertainties for the ALPGEN and SHERPA, and the combined statistical and theoretical uncertainties for BLACKHAT+SHERPA [10].

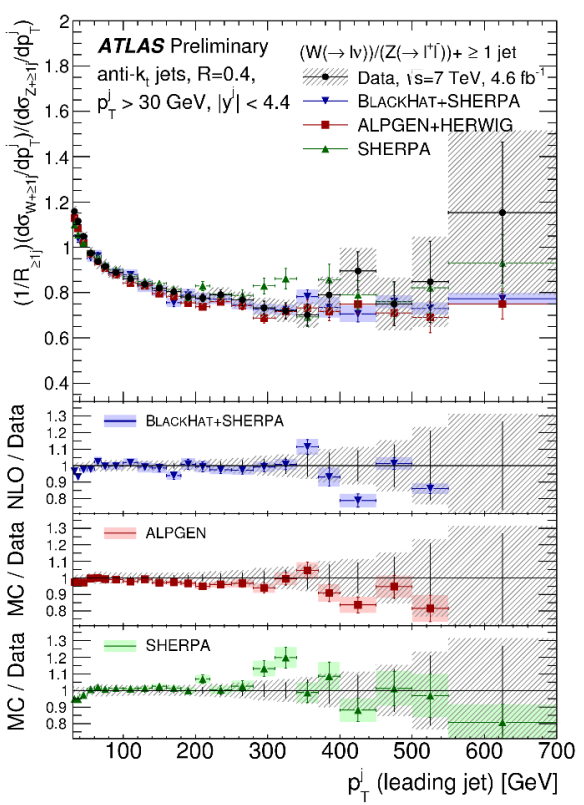

Figure 6. $R_{\text {jets }}$ as a function of leading jet $p_{T}$. This ratio is normalized to the ratio of the $W$ and $Z$ cross sections. The electron and muon channel measurements are combined. Ratios of the simulation to the data are shown in the lower panels. Vertical error bars show the respective statistical uncertainties. The hatched error band shows statical and systematic uncertainties added in quadrature for the data. The solid error bands show statistical uncertainties for the ALPGEN and SHERPA, and the combined statistical and theoretical uncertainties for BLACKHAT+SHERPA [10].

although the SHERPA prediction is about $1 \sigma$ greater than the data at the high jet multiplicity and the ALPGEN prediction shows disagreement in the inclusive ( $\geq 0$ jet) measurement. Figure 6 shows $R_{\text {jets }}$ as a function of the leading jet $p_{T}$ for $N_{\text {jets }} \geq 1$. In the low-transverse momentum region $\left(p_{T}<200\right.$ $\mathrm{GeV})$, the $R_{\text {jets }}$ falls as the leading jet $p_{T}$ increases, indicating that the $p_{T}$ dependence of $W+$ jets and $Z+$ jets events are different. This is due to differences in the masses of the $W$ and the $Z$ bosons, and differences in the vector boson polarisations. The measurement shows reasonable agreement with the theoretical predictions except the high $p_{T}$ region.

\section{$5 W+b$ jets production}

The measurement of $W+b$ jets production allows tests of perturbative QCD in the presence of heavy quarks. In addition, this process is an irreducible background to the Higgs boson associated production process $W H$ with $H \rightarrow b \bar{b}$. Figure 7 shows the unfolded result for the fiducial $W+b$ jets cross section. This measurement requires exactly one $b$-tagged jet to reduce the top quark background 


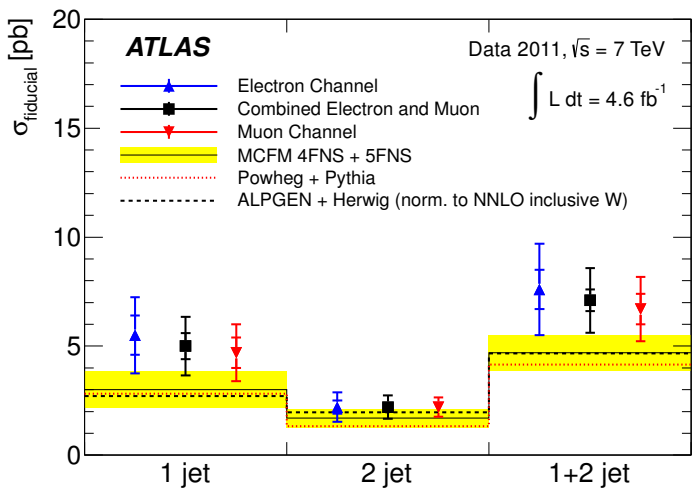

Figure 7. Measured fiducial cross sections in the electron, muon and combined electron and muon channels. Vertical bars show the statistical (inner error bar) and statistical plus systematic (outer error bar) uncertainties. The yellow bands represent the total uncertainty on the prediction [11].

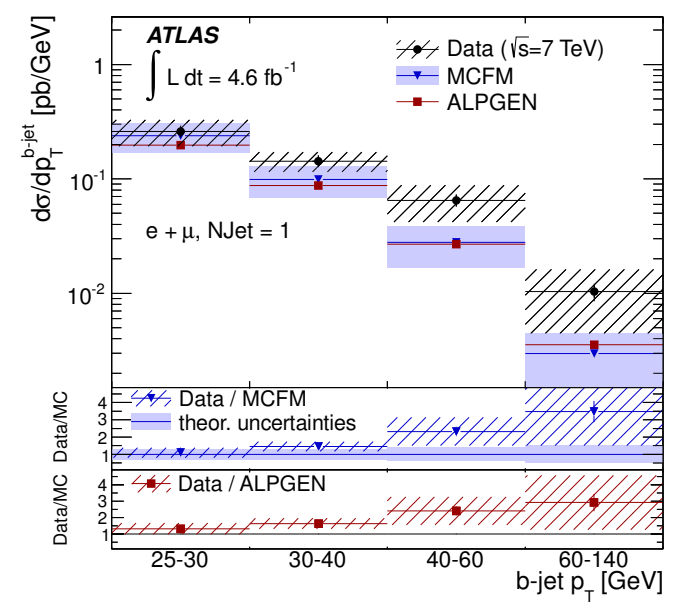

Figure 8. Measured differential $W+b$ jet cross section with statistical (vertical bar) and statistical plus systematic (hatched band) uncertainties as a function of $b$ jet $p_{T}$. The electron and muon channels are combined. Ratios of the simulation to the data are shown in the lower panels [11].

contribution in the 2-jet analysis region. The theoretical predictions for the $W+b$ jets processes with NLO perturbative QCD calculation have recently become available. The data is compared with the NLO predictions calculated by MCFM [23] which is corrected for hadronization and double parton interaction effects, with the POWHEG [24] interfaced to PYTHIA which is corrected for the effect of double parton interactions, and with the prediction from ALPGEN interfaced to HERWIG [25, 26] and JIMMY [27] scaled by the NNLO inclusive $W$ normalization factor. Both the ALPGEN and POWHEG predictions implement a 4-flavour number scheme (4FNS) calculation, while the MCFM prediction includes a 5 -flavour number scheme (5FNS). The 4FNS considers only $u, d, s, c$ quarks in the initial state, while the 5FNS includes $b$ quark in addition. The data is consistent within 1.5 sigma with the NLO prediction in the 1-jet region. The 2-jet region is in good agreement with the theoretical calculations. Figure 8 shows the cross section as a function of $b$ jet $p_{T}$ in the 1 -jet region. The data tends to have larger values compared to the simulation in the large $b$ jet $p_{T}$ region.

\section{$6 Z+b$ jets production}

The measurement of $Z+b$ production constrains the $b$ quark parton distribution function, and this process is an irreducible background to $Z H$ associated Higgs boson production with $H \rightarrow b \bar{b}$. Figure 9 shows the cross section for $Z+\geq 1 b$-jet. The theoretical predictions from MCFM and aMC@NLO in the 5FNS are consistent with the data within the uncertainties. The 5FNS prediction describes $Z+\geq 1 b$-jet well. Figure 10 shows the cross section $\sigma(Z b b)$ as a function of angular separation of the two $b$-jets $(\Delta R(b, b))$. The cross section at low $\Delta R(b, b)$ shows disagreement with the theoretical predictions. 


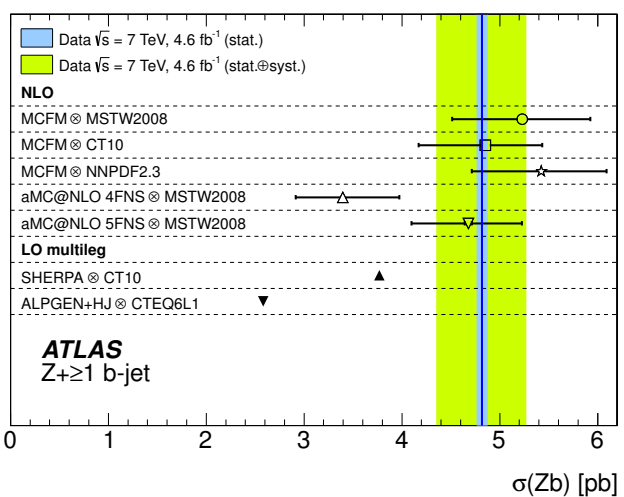

Figure 9. The cross-section of $Z+\geq 1 \quad b$-jet production. The measurement is shown as a vertical blue line with the statistical (blue band) and statistical plus systematic (green band) uncertainties. The uncertainties for theoretical predictions are shown as horizontal bars. Inner bar shows statistical uncertainty, and outer bar shows total uncertainties. Predictions from ALPGEN+HERWIG+JIMMY and SHERPA shows statistical uncertainty only [12].
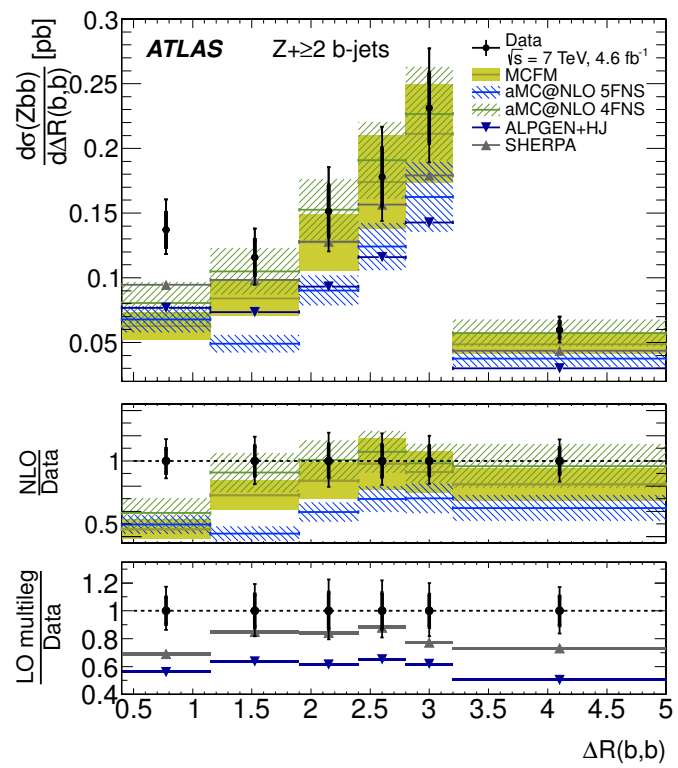

Figure 10. The cross-section of $Z+\geq 2 b$-jets as a function of $\Delta R(b, b)$ with statistical (inner) and total uncertainties (outer bar). The shaded bands represent the total theoretical uncertainties, although the bands on aMC@NLO represent the dominant theoretical scale uncertainty only [12].

\section{$7 W+c$ production}

The $W+c$ production is directory sensitive to the strange PDF of the proton at $x \sim 0.01$. The precise measurement of strange PDF is effective for a test of SU(3) flavour symmetry. Figure 11 shows the cross section ratio $\left(\sigma_{\text {fid }}^{\text {OSSS }}\left(W^{+} D^{(*)-}\right) / \sigma_{\text {fid }}^{\text {OS-SS }}\left(W^{-} D^{(*)+}\right)\right)$. The charm quark is tagged either by the presence of a jet containing a particle semi-leptonically decaying to a muon or by the presence of a charmed hadron reconstructed in the decay modes $D^{+} \rightarrow K^{-} \pi^{+} \pi^{+}$and $D^{*+} \rightarrow D^{0} \pi^{+}$with $D^{0} \rightarrow K^{-} \pi^{+}$, $D^{0} \rightarrow K^{-} \pi^{+} \pi^{0}$ or $D^{0} \rightarrow K^{-} \pi^{+} \pi^{-} \pi^{+}$, and their charge conjugates. To determine the $W+c$ yields, charge correlation between $W$ and $c$ quark is used to reduce $W+c \bar{c}$ background. The data is in good agreement with these theoretical predictions with symmetric $s$ - and $\bar{s}$-quark PDF sets. Figure 12 shows the ratio of strange-to-down sea-quark distributions $r_{s}=0.5(s+\bar{s}) / \bar{d}$ as a function of $x$. ATLAS-epWZ12 is based on the analysis of ATLAS $W$ and $Z$ cross section measurements [28] together with HERA data [29]. ATLAS $W c$-jet/ $W D^{(*)}$ is also combined with HERA data. The ATLAS $W c$-jet/ $W D^{(*)}$ data favors a symmetric light-quark sea over the whole $x$ range relevant to the presented measurement.

\section{Conclusion}

Measurements on the processes of single $W, Z$ boson production in association with jets in $4.6 \mathrm{fb}^{-1}$ of $p p$ collision data at $\sqrt{s}=7 \mathrm{TeV}$ collected with ATLAS detector at LHC are summarized. These measurements constitute important tests of perturbative QCD with light and heavy flavor quarks, constrain the PDF of the proton. These productions become non-negligible background sources for many 


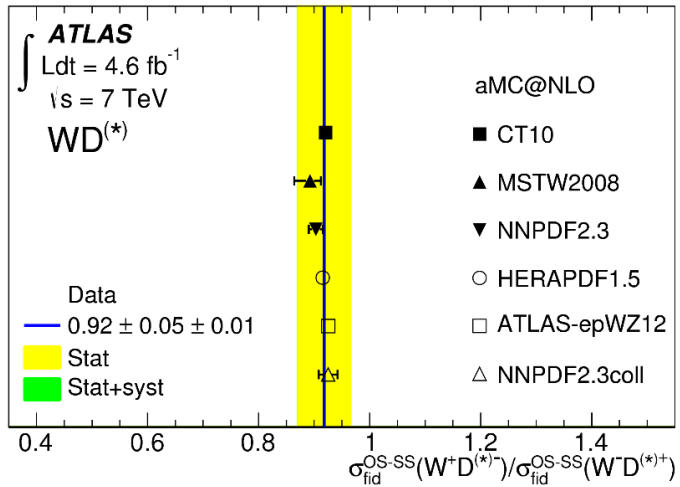

Figure 11. Cross-section ratio between $W^{+} D^{(*)-}$ and $W^{-} D^{(*)+}$. The measurement is shown as a vertical blue line with the statistical (yellow band) and statistical plus systematic (green band) uncertainties [13].

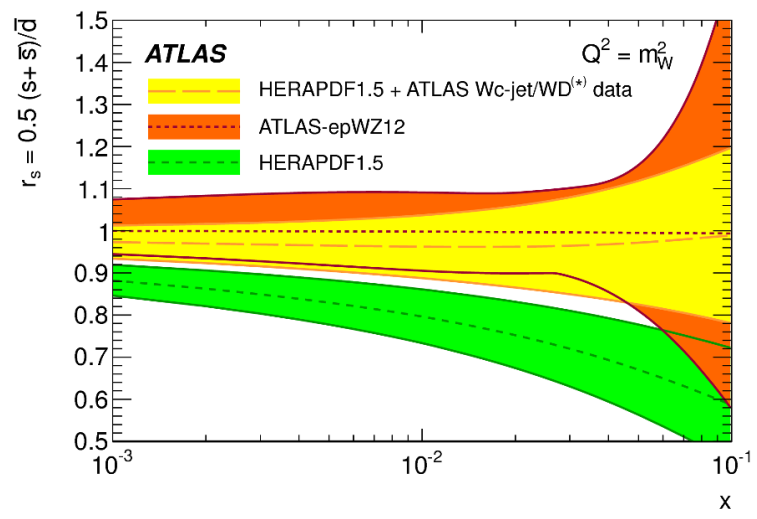

Figure 12. Ratio of strange to down sea quark distributions as a function of $x$. The error band on the ATLAS $W c$-jet $/ W D^{*}$ measurements represents the total uncertainty. The ratio $r_{s}$ is shown at $Q^{2}=m_{w}^{2}[13]$.

physics. In general, good agreement can be seen between the data and typical theoretical predictions. These new measurements highlight the success of recent theoretical advances and the opportunity for further tuning to improve the description of vector boson plus jets.

\section{References}

[1] ATLAS Collaboration, 2008 JINST 3 S08003.

[2] L. Evans and P. Bryant (editors) 2008 JINST 3 S08001.

[3] Z. Bern, et al., Phys. Rev. D88, 014025 (2013).

[4] J.M. Campbell and R. Ellis, Nucl. Phys. Proc. Suppl. 205-206 (2010) 10.

[5] J.M. Campbell and R. Ellis, F. Maltoni, and S. Willenbrock, Phys. Rev. D69 (2004) 074021.

[6] J.M. Campbell and R. Ellis, F. Maltoni, and S. Willenbrock, Phys. Rev. D73 (2006) 054007.

[7] F. Cordero, L. Reina, and D. Wackeroth, Phys. Rev. D80 (2009) 034015.

[8] ATLAS Collaboration, ATLAS-CONF-2014-035, http://cds.cern.ch/record/1735193.

[9] ATLAS Collaboration, JHEP 07 (2013) 032.

[10] ATLAS Collaboration, ATLAS-CONF-2014-034, http://cds.cern.ch/record/1735192.

[11] ATLAS Collaboration, JHEP 06 (2013) 084.

[12] ATLAS Collaboration, http://arxiv.org/abs/1407.3643.

[13] ATLAS Collaboration, JHEP 05 (2014) 068

[14] C. Berger, et al., Phys. Rev. D80 (2009) 074036.

[15] C. Berger, et al., Phys. Rev. D82 (2010) 074002.

[16] C. Berger, et al., Phys. Rev. Lett. 106 (2011) 092001.

[17] T. Gleisberg, et al., JHEP 0902 (2009) 007.

[18] S. Hoeche, F. Krauss, M. Schonherr, F. Siegert, JHEP 1304 (2013) 027.

[19] J.R. Andersen, T. Hapola, J.M. Smillie, JHEP 1209 (2012) 047. 
[20] M. Rubin, G.P. Salam, S. Sapeta, JHEP 1009 (2010) 084

[21] M. L. Mangano, et al., JEHP 0307 (2003) 001. J.R. Andersen, T. Hapola, J.M. Smillie, JHEP 1209 (2012) 047.

[22] S. Frixione and B.R. Webber, JHEP 06 (2002) 029.

[23] J. Campbell, F. Caola, F. Febres Cordero, L. Reina and D. Wackeroth, phys. Rev. D86 (2012) 034021.

[24] S. Alioli, P. Nason, C. Oleari and E. Re, JEHP 06 (2010) 043.

[25] G. Corcella et al., JHEP 01 (2001) 010.

[26] G. Corcella et al., hep-ph/0210213.

[27] J. Butterworth, J.R. Forshaw and M. Seymour, Z. Phys. C72 637.

[28] ALTAS collaboration, Phys. Rev. D 85 (2012) 072994.

[29] H1 and ZEUS collaboration, JHEP 01 (2010) 109 